OPEN ACCESS

Edited by:

Boon-Seng Wong,

Singapore Institute of Technology,

Singapore

Reviewed by:

Hongwei Dong,

Vanderbilt University Medical Center,

United States

Yan Zheng,

Capital Medical University, China

${ }^{*}$ Correspondence:

Hong-Bo Zhao

hzhao2@uky.edu

†These authors have contributed equally to this work

Received: 15 May 2021 Accepted: 09 August 2021 Published: 13 September 2021

Citation:

Mei L, Liu L-M, Chen K and Zhao H-B (2021) Early Functional and Cognitive Declines Measured by Auditory-Evoked Cortical Potentials in Mice With Alzheimer's Disease. Front. Aging Neurosci. 13:710317. doi: 10.3389/fnagi.2021.710317

\section{Early Functional and Cognitive Declines Measured by Auditory-Evoked Cortical Potentials in Mice With Alzheimer's Disease}

\author{
Ling Meit, Li-Man Liu', Kaitian Chen and Hong-Bo Zhao* \\ Department of Otolaryngology, University of Kentucky Medical Center, Lexington, KY, United States
}

Alzheimer's disease (AD) is characterized by a progressive loss of memory and cognitive decline. However, the assessment of AD-associated functional and cognitive changes is still a big challenge. Auditory-evoked cortical potential (AECP) is an event-related potential reflecting not only neural activation in the auditory cortex (AC) but also cognitive activity in the brain. In this study, we used the subdermal needle electrodes with the same electrode setting as the auditory brainstem response (ABR) recording and recorded AECP in normal aging CBA/CaJ mice and APP/PS1 AD mice. AECP in mice usually appeared as three positive peaks, i.e., P1, P2, and P3, and three corresponding negative peaks, i.e., N1, N2, and N3. In normal aging CBA mice, the early sensory peaks $\mathrm{P} 1, \mathrm{~N} 1$, and P2 were reduced as age increased, whereas the later cognitive peaks N2, P3, and N3 were increased or had no changes with aging. Moreover, the latency of the $\mathrm{P} 1$ peak was increased as age increased, although the latencies of later peaks had a significant reduction with aging. In $A D$ mice, peak $\mathrm{P} 1$ was significantly reduced in comparison with wild-type (WT) littermates at young ages, proceeding AD phenotype presentation. In particular, the later cognitive peak P3 was diminished after 3 months old, different from the normal aging effect. However, the latencies of AECP peaks in $A D$ mice generally had no significant delay or changes with aging. Finally, consistent with AECP changes, the accumulation of amyloid precursor protein (APP) at the AC was visible in AD mice as early as 2 months old. These data suggest that AECP could serve as an early, non-invasive, and objective biomarker for detecting AD and AD-related dementia (ADRD).

Keywords: auditory-evoked cortical potential, auditory cortex, Alzheimer's disease, dementia, hearing loss, cognitive decline, mouse model, event-related potential

\section{INTRODUCTION}

Alzheimer's disease (AD) is a progressive neurodegenerative disease, characterized by a progressive loss of memory and dementia. Over the last two decades, the prevalence of $\mathrm{AD}$ and $\mathrm{AD}$-related dementia (ADRD) has been rapidly increased. It is prospected that there will be 150 million $\mathrm{AD}$ patients by the year 2050, almost triple the population in 2018 (Hebert et al., 2013; Patterson, 2018). 
This will produce a heavy economic and social burden. Early detection is critical for the prevention and interventions of this common neurodegenerative disease. It is estimated that early diagnosis and interventions delaying the onset of dementia by even 1 year could decrease the worldwide prevalence of dementia by $10 \%$ (Patterson, 2018). However, since the lack of early, reliable $\mathrm{AD}$ biomarkers, early detection of $\mathrm{AD}$ is still a big challenge. In particular, it lacks objective biomarkers to detect cognitive decline, which is the most common and predominant symptom of $\mathrm{AD}$, at the early stage.

Recently, increasing evidence suggests that defects in sensory systems, including the olfactory, visual, and auditory systems, are highly associated with AD progression (Murphy, 2019; Rochoy et al., 2019). AD pathology could appear in sensoryassociated areas before appearing in regions involving memory, such as the entorhinal and hippocampal areas (Gates et al., 2002; Murphy, 2019). These neuropathological changes in the sensoryassociated areas could also occur before cognitive symptoms become apparent. Hearing is an important neural sense. AD can cause hearing loss, and hearing loss can in turn accelerate $\mathrm{AD}$ and dementia development (Morrison et al., 2018; Griffiths et al., 2020). Our previous study demonstrated that auditory brainstem response (ABR), which reflects the function and integrity of the auditory pathway in the brainstem, had a reduction in APP/PS1 $\mathrm{AD}$ mice at the age of 3 months old (Liu et al., 2020), prior to the occurrence of typical $\mathrm{AD}$ phenotypes, such as spatial learning deficit, in this $\mathrm{AD}$ mouse model by the age of 6-7 months old (Reiserer et al., 2007; Ordóñez-Gutiérrez et al., 2015, 2016). Recent studies also found that deficits in auditory gap detection could occur in young 5xFAD mice (Kaylegian et al., 2019; Weible et al., 2020). These studies suggest that AD could cause hearing function decline at the early stage and that hearing function tests could provide important information for $\mathrm{AD}$ development at the early stage.

Auditory-evoked cortex potential (AECP) is an event-related potential evoked by auditory stimulation and reflects the neural activity in the auditory cortex (AC) and the related brain areas. In humans, AECP consists of several waveforms and are named as P1 (P50), N1 (N100), P2 (P200), N2 (N250), P3 (P300), and P4 (P600) based on their latencies (Swords et al., 2018). Early waveforms (i.e., P1 and P2) are "sensory," largely depending on the stimulus and activities in the AC; later waveforms (i.e., P3 and P4) are "cognitive" reflecting cognitive processing (Sur and Sinha, 2009). Thus, unlike ABR, the AECP can reflect not only the neural activities in the AC but also the cognitive activities in the brain.

In previous studies, auditory-evoked potentials were recorded in the cortex, hippocampus, and other brain areas with implanted electrodes in AD animal models (Wang et al., 2003; Stoiljkovic et al., 2019) or amyloid- $\beta$ (A $\beta$ ) injected in the rat and mouse brain at different ages (Hidisoglu and Yargicoglu, 2020; Kim et al., 2020). In general, auditory-evoked potentials in the AC and other brain cortexes were reduced in $\mathrm{AD}$ or amyloidinjected animals. However, there were conflicting results in the literature (Gurevicius et al., 2013). AD-induced auditory-evoked potential changes in the brain and their dynamic changes in $\mathrm{AD}$ development still remain largely undetermined. In this study, we systematically recorded AECP in APP/PS1 AD mice to assess
$\mathrm{AD}$-induced functional changes in the $\mathrm{AC}$ and brain with aging. We found that AECP in $\mathrm{AD}$ mice had a significant reduction at young ages. In particular, later cognitive peak $\mathrm{P} 3$ in $\mathrm{AD}$ mice was diminished, different from the normal aging effect. Our study suggests that AECP recording could serve as an early non-invasive biomarker to assess $\mathrm{AD}$ and $\mathrm{ADRD}$ development.

\section{MATERIALS AND METHODS}

\section{APP/PS1 AD Mice and CBA/CaJ Normal Aging Mice}

APP/PS1 transgenic mice were purchased from Jackson Laboratory, United States (Stock No: 004462, mixed C57BL/6; $\mathrm{C} 3 \mathrm{H}$ genetic background). Since the $\mathrm{C} 57 \mathrm{BL} / 6$ genetic background is associated with age-related hearing loss, we crossed APP/PS1 mice with CBA/CaJ mouse strain (Stock No: 00654, Jackson Laboratory, Bar Harbor, ME, United States), which has no apparent age-related hearing loss (Zheng et al., 1999), for four generations. APP/PS1 transgenic mice possess both chimeric mouse/human amyloid precursor protein (APP) (Mo/HuAPP695swe) and mutant human presenilin 1 (PS1-dE9) (Reiserer et al., 2007). According to the technical notice of Jackson Laboratory, APP/PS1 transgenes were detected by PCR amplification of tail genomic DNA using the following primers: wild-type (WT) forward: $5^{\prime}$-GTG TGA TCC ATT CCA TCA GC-3'; common: 5'-GGA TCT CTG AGG GGT CCA GT-3'; mutant forward: 5'-ATG GTA GAG TAA GCG AGA ACA CG-3'. Mutant and WT mice generated 142 and 265 bps bands, respectively. WT littermates served as controls. Both genders were used in the experiment. CBA/CAJ mice were also used for the normal aging studies. All experiments and procedures were approved and conducted in accordance with the policies of the University of Kentucky Animal Care and Use Committee (approved protocol: UK\#2020-3524).

\section{Auditory-Evoked Cortical Potential Recording}

Auditory-evoked cortical potential was recorded in a double-wall sound isolated chamber by use of a Tucker-Davis R3 system with an ES-1 high-frequency speaker (Tucker-Davis Tech., Alachua, FL, United States). Mice were anesthetized by intraperitoneal injection with a mixture of ketamine and xylazine $(8.5 \mathrm{ml}$ saline $+1 \mathrm{ml}$ ketamine $+0.55 \mathrm{ml}$ xylazine, $0.1 \mathrm{ml} / 10 \mathrm{~g}$ ). Body temperature was maintained at $37-38^{\circ} \mathrm{C}$ by placing anesthetized mice on an isothermal pad. As the same as ABR recording (Liu et al., 2020), two subdermal needle electrodes were punched into the head skin at the vertex and ventrolaterally to the right or left ear. The ground needle electrode was inserted into the right leg. Cortical potentials were evoked by clicks ( $85 \mathrm{~dB}$ SPL) in alternative polarity with a stimulating rate of $1 / \mathrm{s}$. The recording time window was $800 \mathrm{~ms}$, starting from the beginning of the stimulation. The signal was amplified by 50,000 and averaged by 200 times. The recording filter was set at $0-3 \mathrm{kHz}$. The signal recording was performed by BioSigRP software (Tucker-Davis Tech.). The collected signals were further filtered in offline with 
a build-in 10-order digital Gaussian filter with $12.5 \mathrm{~Hz}$ of central frequency and a bandwidth of 2 octaves for analysis.

\section{Brain Slide Preparation and Immunofluorescent Staining}

Mice were anesthetized with ketamine as described above and intracardially perfused with $4 \%$ paraformaldehyde for $15-$ 20 min. After decapitation, the mouse skull was opened. The brain was carefully removed and incubated overnight in $4 \%$ paraformaldehyde for postfixation. Following infiltration with $30 \%$ glucose for 3 days with changing fresh solution at each day, the brain was embedded in OCT (Cat \# 4583, Sakura Finetek USA, Inc., Torrance, CA, United States) at room temperature for 1 day. Then, the embedded brain was frozen at $-80^{\circ} \mathrm{C}$ overnight and cut $20 \mu \mathrm{m}$ thick at $-22-24^{\circ} \mathrm{C}$ by a cryostat (Thermo Electron Corp., Waltham, MA, United States). The tissue sections were directly mounted onto glass slides for staining and storage.

Immunofluorescent staining was performed as described in our previous reports (Zhao and $\mathrm{Yu}, 2006$; Wang et al., 2009). After $1 \mathrm{~h}$ of incubation with the blocking solution ( $10 \%$ goat serum and $1 \%$ BSA in PBS) with $0.5 \%$ Triton X100 at room temperature, the brain slides were incubated with primary antibody rabbit monoclonal anti-APP (clone Y188) (\#AB32136, Abcam, Waltham, MA, United States; 1:500) in blocking solution at $4^{\circ} \mathrm{C}$ overnight. After washout with PBS three times, the brain slides were incubated at room temperature with corresponding secondary antibody conjugated with Alexa Fluor568 (Thermo Fisher Scientific, MA, United States) for $2 \mathrm{~h}$. At the last $15 \mathrm{~min}, 4^{\prime}, 6$-diamidino-2-phenylindole (DAPI, $0.1 \mathrm{mg} / \mathrm{ml}$, D1306; Molecular Probes) was added into the incubation solution to visualize the cell nuclei. After completely washing out the secondary antibodies with PBS, the sections were mounted with a fluorescence mounting medium H-1000, Vector Lab, CA, United States) and observed under a Nikon A1R confocal microscope system. The $\mathrm{AD}$ and $\mathrm{WT}$ mouse sections were simultaneously stained and observed with the same parameters.

\section{Data Processing, Statistical Analysis, and Reproducibility}

The amplitude and latency of peaks in AECP were measured by a house-made program with MATLAB (MathWorks Inc., MA, United States). Data were plotted by SigmaPlot and statistically analyzed by SPSS v25.0 (SPSS Inc., Chicago, IL, United States). Data were expressed as mean \pm SEM. Student's $t$-tests were performed between APP and WT groups at the defined age. When three or more groups were compared, one-way ANOVA with Bonferroni post hoc test was used. The threshold for significance was $\alpha=0.05$. The AECP was recorded from mice generated by more than 15 different breeding pairs.

\section{RESULTS}

\section{AECP in Mice}

Figure 1 shows AECP recorded from mice. The AECP recorded from mice usually appeared as three positive peaks, i.e., P1,
$\mathrm{P} 2$, and $\mathrm{P} 3$, and three corresponding negative peaks, i.e., N1, N2, and N3 (Figure 1). Like AECP recorded from humans, both early sensory peaks (P1, N1, and P2) and later cognitive waves ( $\mathrm{P} 3$ and N3) were visible in the recording. However, the later cognitive wave P3 in mice was not clearly visible until the age of 3 months old (Figures 1A,B). At 6 months old, $\mathrm{AECP}$ in mice was well developed, and $\mathrm{P} 1, \mathrm{P} 2$, and $\mathrm{P} 3$ peaks were clearly visible (Figure 1C). The latencies of peaks P1, P2, and $\mathrm{P} 3$ were $13.5 \pm 1.89,67.5 \pm 2.74$, and $128.1 \pm 6.76 \mathrm{~ms}$, respectively (Figure $2 \mathrm{~A}$ ).

\section{Normal Aging Changes of AECP in CBA/CaJ Mice}

We further investigated the effect of normal aging on AECP. $\mathrm{CBA} / \mathrm{CAJ}$ mice, which have no apparent age-related hearing loss (Zheng et al., 1999), were used. Figure 1 shows that AECPs in CBA mice were reduced with increased age. The amplitudes of early peaks (i.e., P1, N1, and P2) were $4.66 \pm 0.70$, $-4.73 \pm 0.71$, and $3.15 \pm 0.75 \mu \mathrm{V}$, respectively, at 12 months old and $3.39 \pm 0.77,-3.98 \pm 0.78$, and $3.41 \pm 0.86 \mu \mathrm{V}$, respectively, at 18 months old (Figures 2C,D). In comparison with those at the age of 3 months, the amplitudes of $\mathrm{P} 1, \mathrm{~N} 1$, and $\mathrm{P} 2$ at 12 months old had a significant reduction $(p=0.003-0.022, t$-test, twotailed). However, the peak P3 was significantly increased from $2.03 \pm 0.31 \mu \mathrm{V}$ at 3 months old to $3.47 \pm 0.56 \mu \mathrm{V}$ at 12 months old ( $p=0.037, t$-test, two-tailed) (Figure 2C). The amplitudes of later peaks N2 and N3 had no significant changes with aging (Figure 2D).

The latency of P1 peak had a significant increase at 6 and 12 months ( $p=0.014$ and 0.045 , respectively, $t$-test, two-tailed) (Figure 2B). However, there was no significant change in the N1 latency with aging; the latencies of peaks $\mathrm{P} 2, \mathrm{~N} 2, \mathrm{P} 3$, and $\mathrm{N} 3$ were significantly reduced ( $p=0.004-0.045, t$-test, two-tailed) at 6 and 12 months old but had no significant changes at 18 months old in comparison with them at 3 months old (Figure 2B).

\section{AECP in APP/PS1 AD Mice}

Figure 3 shows AECP traces recorded from APP/PS1 AD mice and WT littermates at different ages. In general, P1 and P2 in $\mathrm{AECP}$ in $\mathrm{AD}$ mice appeared smaller than those in WT littermate mice at young ages. At the age of 1-3 months old, there was a notch at the descending side of peak P2 in AD mice (indicated by blue arrows in Figures 3A-C). P3 is clearly visible in WT mice after 4 months old (indicated by red arrows in Figures 3D-I), but small or invisible in AD mice (Figures 3D-I). Later negative peaks of $\mathrm{N} 2$ and $\mathrm{N} 3$ were also small or invisible in $\mathrm{AD}$ mice (Figures 3D-I).

\section{Changes of AECP in APP/PS1 AD Mice With Aging}

To quantify changes of AECP in AD mice, we measured peaks of AECP at different ages (Figure 4). Quantitative measurement shows that the early P1 peak in AD mice at 3-7 months old was significantly reduced in comparison with those in WT mice ( $p=0.014-0.046, t$-test, two-tailed) (Figure 4A). The amplitudes of later cognitive $\mathrm{P} 3$ peak in $\mathrm{AD}$ mice were also significantly 

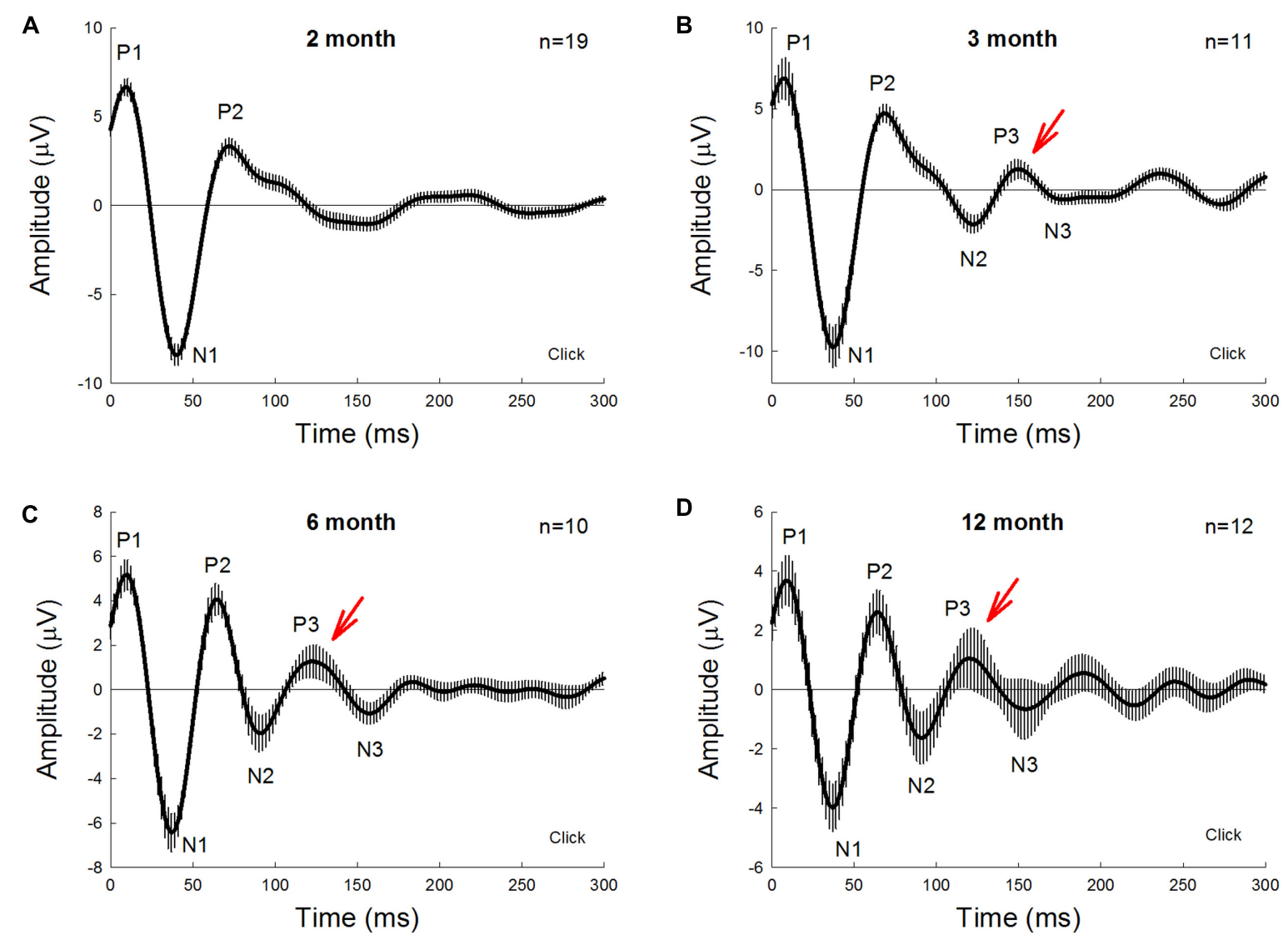

FIGURE 1 | Auditory-evoked cortex potential (AECP) in CBA/CaJ mice at different ages. AECP was evoked by a click stimulus (85 dB SPL) in alternative polarity. Traces were averaged from the recorded mice with both genders. Error bars represent SEM. Mouse age is indicated in each panel. $N$ is the mouse number. AECP in mice has three positive peaks, i.e., P1, P2, and P3, and three corresponding negative N1, N2, and N3 peaks. Red arrows indicate P3 peak that is clearly visible in $\mathrm{AECP}$ at 3 months old and afterward.

smaller than WT mice after 3 months old $(p=0.049-0.005$, $t$-test, two-tailed) (Figure 4E). However, the peak N1 and P2 in $\mathrm{AD}$ mice had no significant changes except 4 months old, at which they had a significant reduction in comparison with WT mice ( $p=0.046-0.048$, $t$-test, two-tailed) (Figures 4B,C). Later peaks N2 and N3 in AD mice also had no significant reduction in comparison with WT mice except 10-12 months old, at which the $\mathrm{N} 3$ peak in AD mice was significantly reduced $(p=0.024-0.047$, $t$-test, two-tailed) (Figure 4F).

The latencies of AECP peaks in $\mathrm{AD}$ mice usually had no significant changes or appeared shortening except a few time points (Figure 5). For example, there was no significant difference in latencies of positive peaks (P1, P2, and P3) between AD and WT mice except 3 months old (Figure 5A), at which the latency of $\mathrm{P} 1$ peak in AD mice was significantly shorter than that in WT mice ( $p=0.0007, t$-test, two-tailed). For negative peaks, the latency of peak $\mathrm{N} 1$ in $\mathrm{AD}$ mice appeared shorter than that in WT mice ( $p=0.0003-0.02, t$-test, two-tailed) at 1,3 , and 6 months old (Figure 5B). The latency of N3 peak in AD mice also appeared shorter than those in WT mice $(p=0.012-0.049$, $t$-test, two-tailed) at 10-12 months old (Figure 5F). However, the latency of peak $\mathrm{N} 2$ in $\mathrm{AD}$ mice appeared longer than that in WT mice ( $p=0.049$, $t$-test, two-tailed) at 12 months old (Figure 5D).

\section{Different Changes of AECP in AD Mice and CBA/CaJ Mice With Aging}

We further examined AECP changes in AD mice, WT littermate mice, and CBA mice at different ages (Figure 6). Changes of AECP in WT littermates and CBA mice with normal aging were similar. However, $\mathrm{P} 1$ peak in $\mathrm{AD}$ mice had a significant reduction at 3 and 6 months old ( $p=0.024-0.035$, one-way ANOVA with a Bonferroni correction) (Figure 6A). Also, P3 peak in AD mice at 3-12 months old was significantly reduced in comparison with that in WT littermates and CBA mice $(p=0.0005-0.036$, oneway ANOVA with a Bonferroni correction) (Figure 6E). The later peaks of N2 and N3 in AD mice were also significantly reduced at 12 months old ( $p=0.004-0.024$, one-way ANOVA with a Bonferroni correction) (Figures 6D,F). However, there were no significant differences in $\mathrm{N} 1$ and $\mathrm{P} 2$ peaks among AD mice, WT littermates, and CBA mice (Figures 6B,C).

To assess the aging effect on AECP in $\mathrm{AD}$ mice, WT littermates, and CBA mice, the amplitudes of AEACP peaks at 

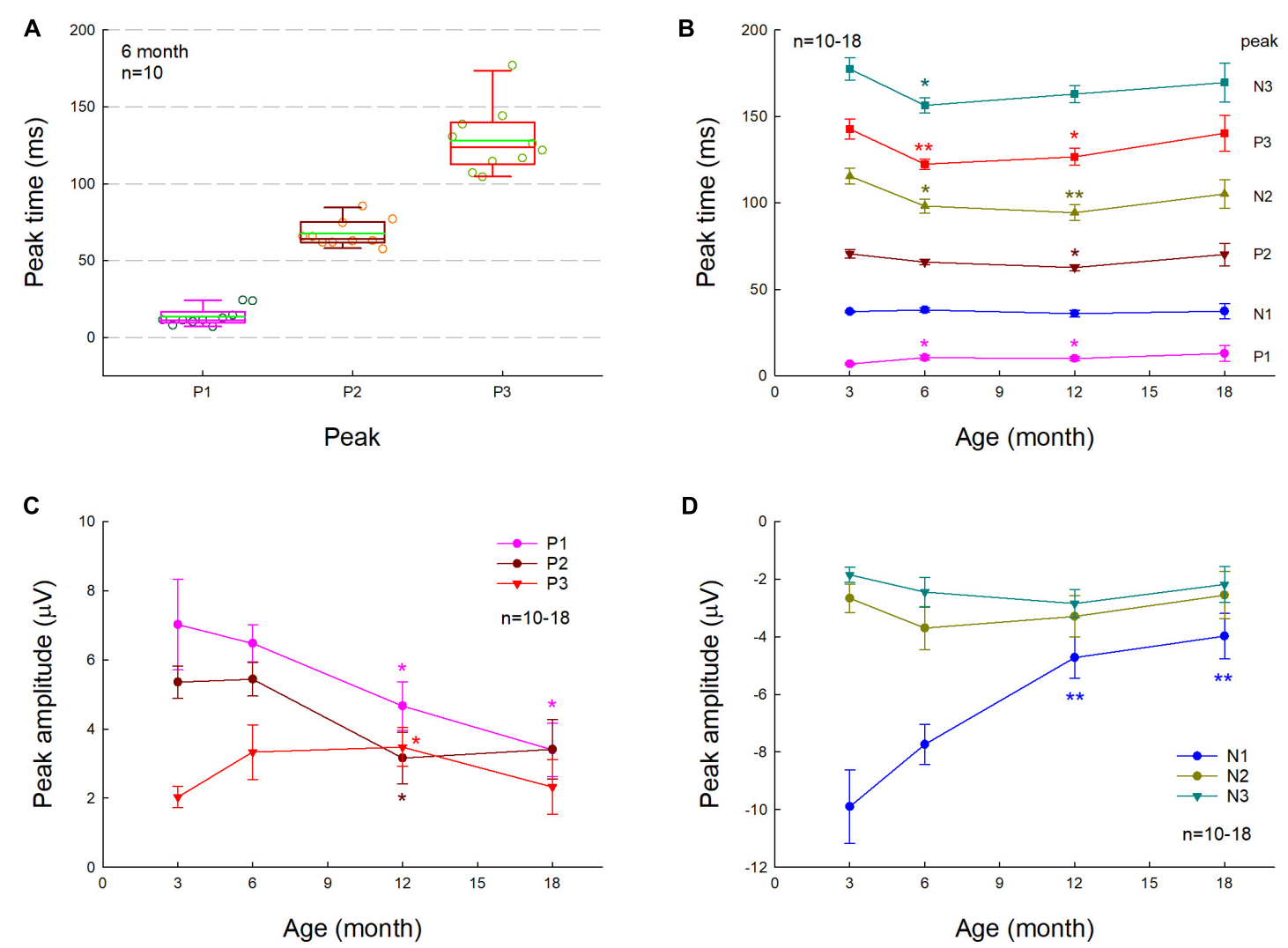

FIGURE 2 | Changes of AECP in normal aging CBA/CaJ mice. $N$ is the mouse number. (A) P1, P2, and P3 peak times at the age of 6 months old. The green line in the box represents the mean level. (B) Changes of AECP peak times in normal aging. The statistical $t$-tests were performed by comparing peak times at the defined age to that at 3 months old in each peak. (C,D) Changes of peak amplitudes with aging. The amplitudes of early peaks P1, N1, and P2 in AECP are decreased with aging, whereas the peak amplitudes of later waveforms N2, P3, and N3 are increased or have no significant change with aging. The statistical analyses were performed by comparing peak amplitudes at the defined age to that at age of 3 months old in each peak. ${ }^{*} p<0.05$, ${ }^{\star \star} p<0.01, t$-test, two-tailed.

different ages were normalized to those at 3 months old in each group. Figure 7 shows that the early sensory P1, N1, and P2 peaks in CBA mice and WT littermates were decreased as age increased and had a significant reduction at the age of 12 months old in comparison with those at 3 months old $(p=0.0003-0.02, t$-test, two-tailed). However, the peak P1, N1, and P2 in AD mice had no significant reduction or change with aging (Figures $7 \mathrm{~A}-\mathrm{C}$ ). For later cognitive peaks, N2, P3, and N3 in CBA mice and WT littermates appeared to increase with aging (Figures 7D-F). In comparison with 3 months old, the increases of N2 in WT littermates at 12 months, $\mathrm{P} 3$ peak in $\mathrm{CAB}$ mice at 12 months, and N3 peak in WT littermates at 6 months old were significant ( $p=0.023-0.037$, $t$-test, two-tailed). However, AECP in AD mice had no significant changes as age increased, except N3 peak at 12 months was significantly decreased in comparison with that at 6 months old ( $p=0.006$, $t$-test, two-tailed) (Figure 7F).

\section{Expression of APP at the Auditory Cortex in APP/PS1 AD Mice}

Since APP/PS1 transgenic mice possess both chimeric mouse/human APP (Mo/HuAPP695swe) and mutant human presenilin 1 (PS1-dE9) (Reiserer et al., 2007), we used anti-APP antibody (clone Y188, aa750 to the C-terminus) (\#AB32136, Abcam, United States) for immunofluorescent staining. This antibody can also react with gamma secretase fragments and C-terminal fragments. Immunofluorescent staining shows that positive labeling was visible at the $\mathrm{AC}$ in $\mathrm{APP} / \mathrm{PS} 1 \mathrm{AD}$ mice at 2 months old (Figures 8B,E), consistent with early changes in the AECP recording. However, there was no labeling visible in WT control mice at the same age (Figure 8C). As age increased, the deposition of labeling in the AC was increased. At the age of 1 year old, the typical accumulating pattern of plaque deposition was clearly visible in the AC (Figures 8A,D).

\section{DISCUSSION}

In this study, we used the same subdermal needle electrodes and setting as the ABR recording and recorded AECP in mice and characterized changes of AECP in APP/PS1 AD mice and normal aging $\mathrm{CBA} / \mathrm{CaJ}$ mice. Like the AECP recorded from humans, both early sensory and later cognitive waves were visible in the recorded mouse AECP, which usually presented three waveforms (Figure 1). The later cognitive peak $\mathrm{P} 3$ was not clearly visible until 3 months old (Figure 1B). As age increased, the early peaks (P1, 

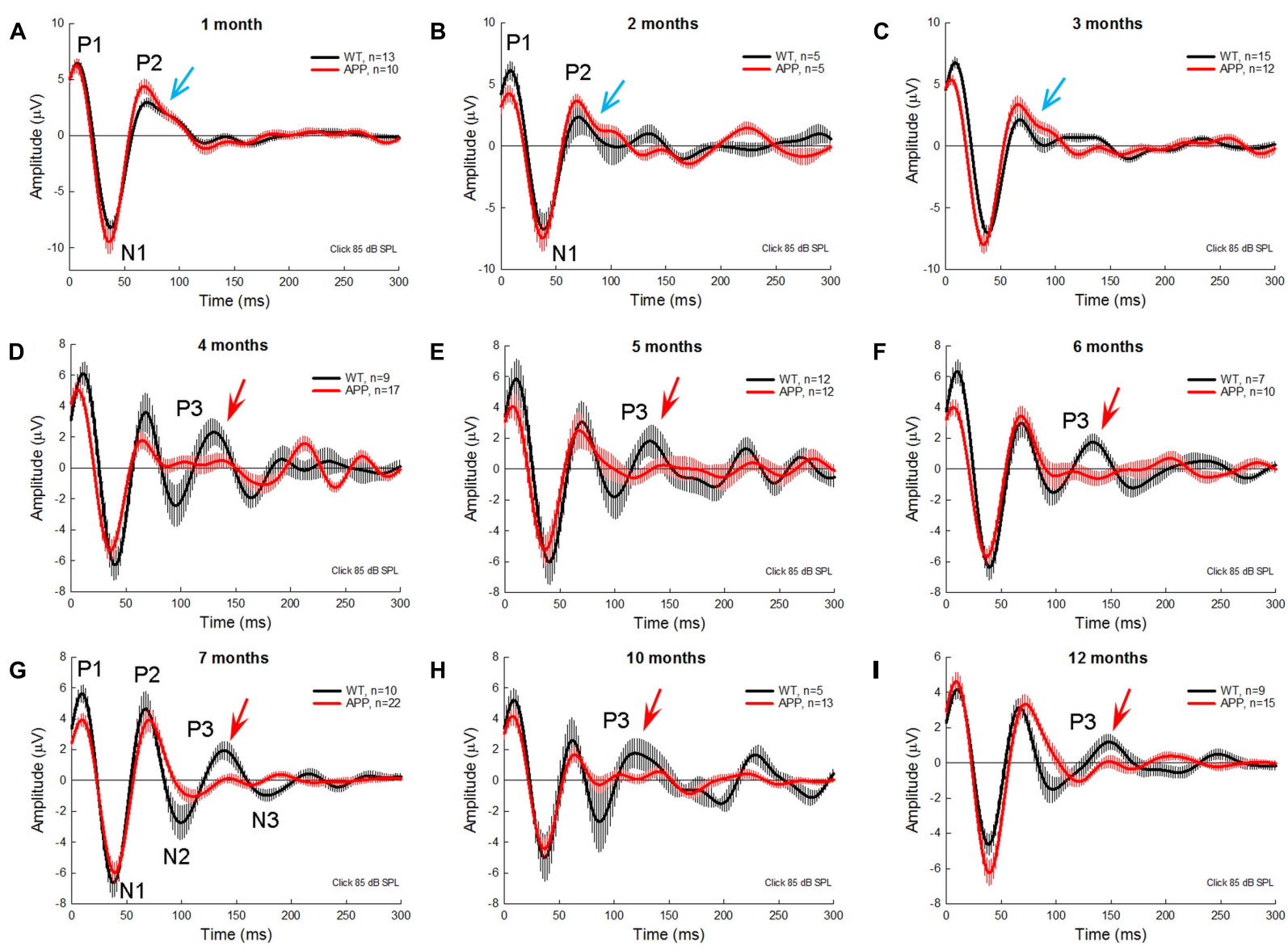

FIGURE 3 | AECP traces were recorded from APP/PS1 AD mice at different ages. WT littermates served as control. Red and black traces represent averaged AECP traces recorded from APP/PS1 AD mice and WT mice, respectively. Error bars represent SEM. Mouse age is indicated in each penal. N is the mouse number. Blue arrows indicate a notch at the descending side of peak P2 in AD mice at 1-3 months old. Red arrows indicate the P3 peak clearly visible in WT mice but not in AD mice after 3 months old.

$\mathrm{N} 1$, and P2) in AECP were reduced, while later peaks (i.e., N2, P3, and N3) were increased or had no changes (Figures 1, 2, 7). In APP/PS1 AD mice, $\mathrm{P} 1$ peak at young ages $(<8$ months old) appeared smaller than those in WT mice (Figures 3, 4, 6). In particular, the later cognitive peak $\mathrm{P} 3$ in $\mathrm{AD}$ mice was diminished (Figures 3, 4, 6). As age increased, there were no significant changes in AECP in AD mice except peak N3 at 1 year old (Figure 7). Also, there was no significant peak delay in AD mice except peak N2 at 1 year old (Figure 5). Finally, consistent with early changes of AECP in young ages, intensive labeling for APP was visible in the $\mathrm{AC}$ of $\mathrm{AD}$ mice at 2 months old (Figure 8). These data suggest that AECP could serve as an early, noninvasive biomarker to detect and assess $\mathrm{AD}$ development and progression.

In previous studies, various auditory-evoked potentials were recorded from animals with implanted electrodes in the brain (Wang et al., 2003; Gurevicius et al., 2013; Kim et al., 2020). However, different from AECP recorded from humans, only early sensory peaks P1 and P2 were visible in those animal recordings; later cognitive peaks were usually missing or invisible even in the recording in the waking animals. In this study, we used subdermal needle electrodes with the same electrode configuration (or setting) as $\mathrm{ABR}$ recording and recorded AECP from ketamine-anesthetized mice. The recorded AECP waveforms are like AECP recorded from humans (Golob et al., 2009; Swords et al., 2018); both early sensory and later cognitive peaks were visible in the recording (Figures 1, 3). However, in comparison with the AECP recorded from humans, the AECP in mice appeared only as three waveforms (Figure 1). Also, the latencies of P1, P2, and P3 in mice were in the range of 10-15, 6570, and 110-150 ms, respectively (Figures 2A,B), and appeared much shorter than humans (Golob et al., 2009; Swords et al., 2018). This may be due to the mouse brain size is small and the neural interactions are less than humans. In addition, P3 in mice was not well developed until 3 months old (Figure 1). As mentioned above, later waveforms in AECP are associated with cognitive processing (Sur and Sinha, 2009). Specifically, P3 is dependent on internal thought process and is considered to be mainly generated by the hippocampus where short-term memory is stored. These data suggest that such cognition-associated neural activity in mice may need to take a longer time for maturation in the development. These data are also consistent 

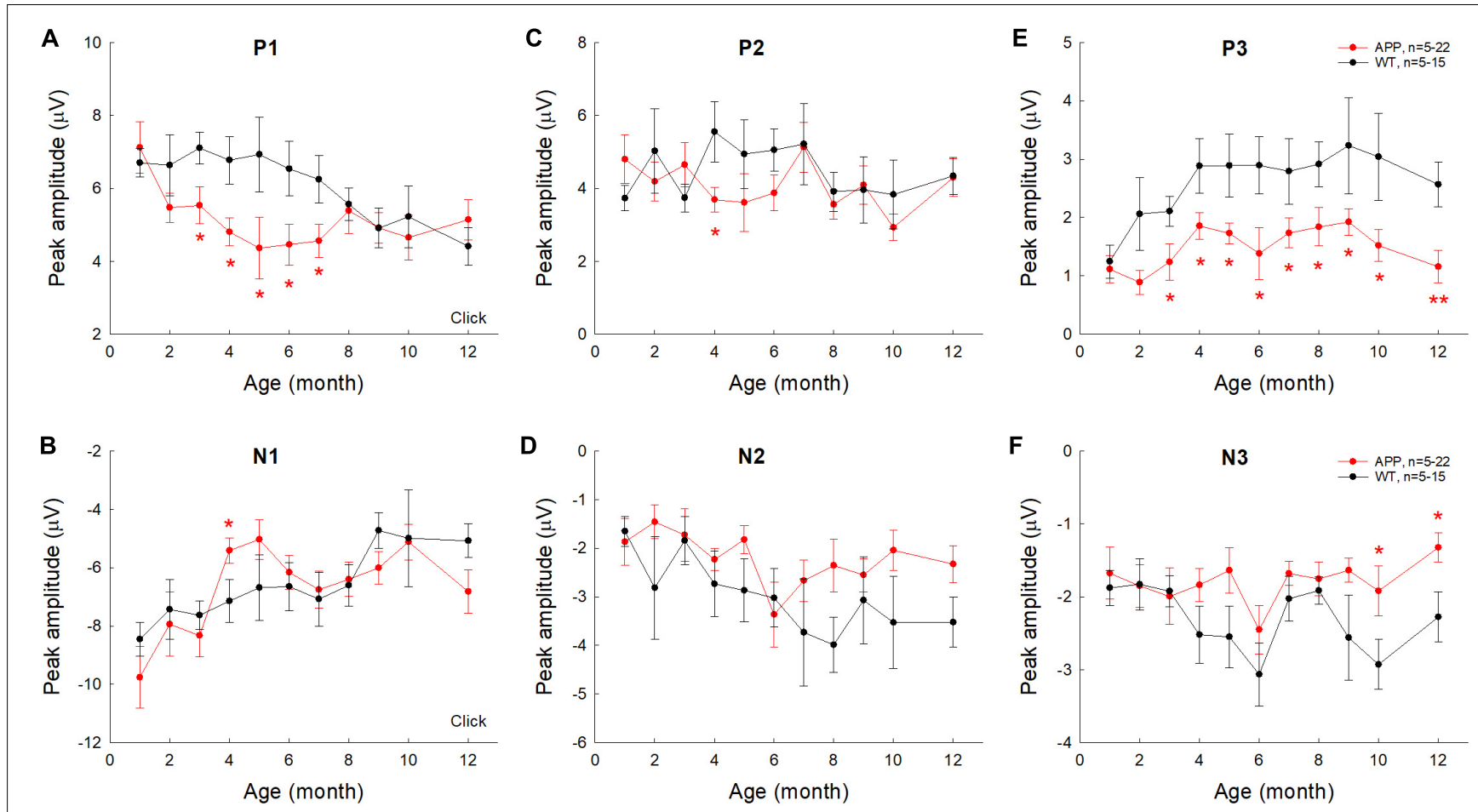

FIGURE 4 | Changes of AECP peaks in APP/PS1 AD mice with aging. WT littermates served as control. Peaks of AECP recorded from each mouse were measured and averaged. Peak name is presented at the top middle place in each panel. $\mathrm{N}$ is the mouse number. The amplitudes of peak P3 in AD mice are significantly less than those in WT mice after 3 months old. ${ }^{*} p<0.05,{ }^{* *} p<0.01, t$-test, two-tailed.

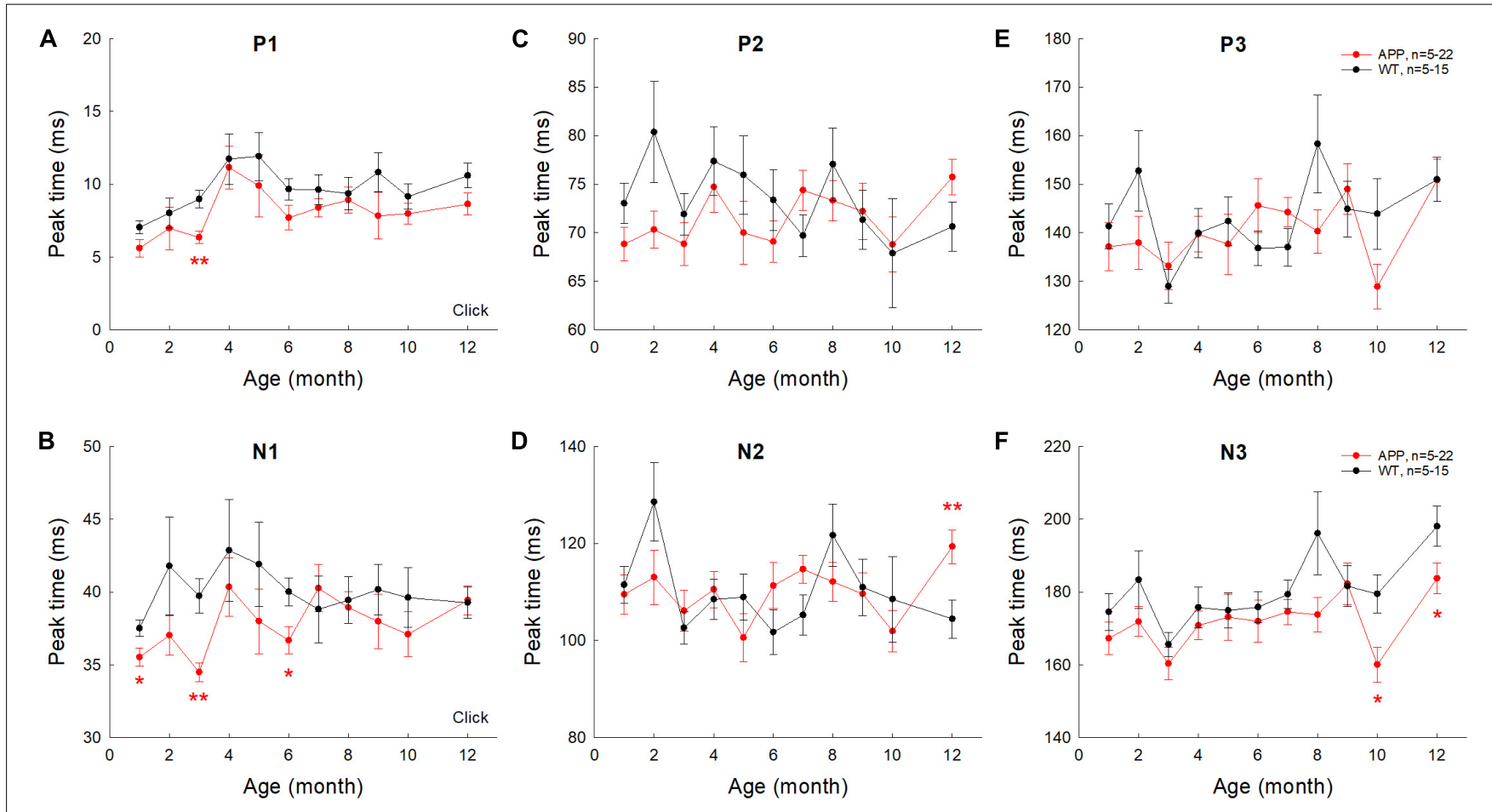

FIGURE 5 | Changes of AECP peak times in APP/PS1 AD mice with aging. Peak name is presented at the top middle place in each panel. $\mathrm{N}$ is the mouse number. The peak times in AD mice were compared with those in WT littermates at the same age. ${ }^{*} p<0.05,{ }^{* \star} p<0.01, t$-test, two-tailed. 

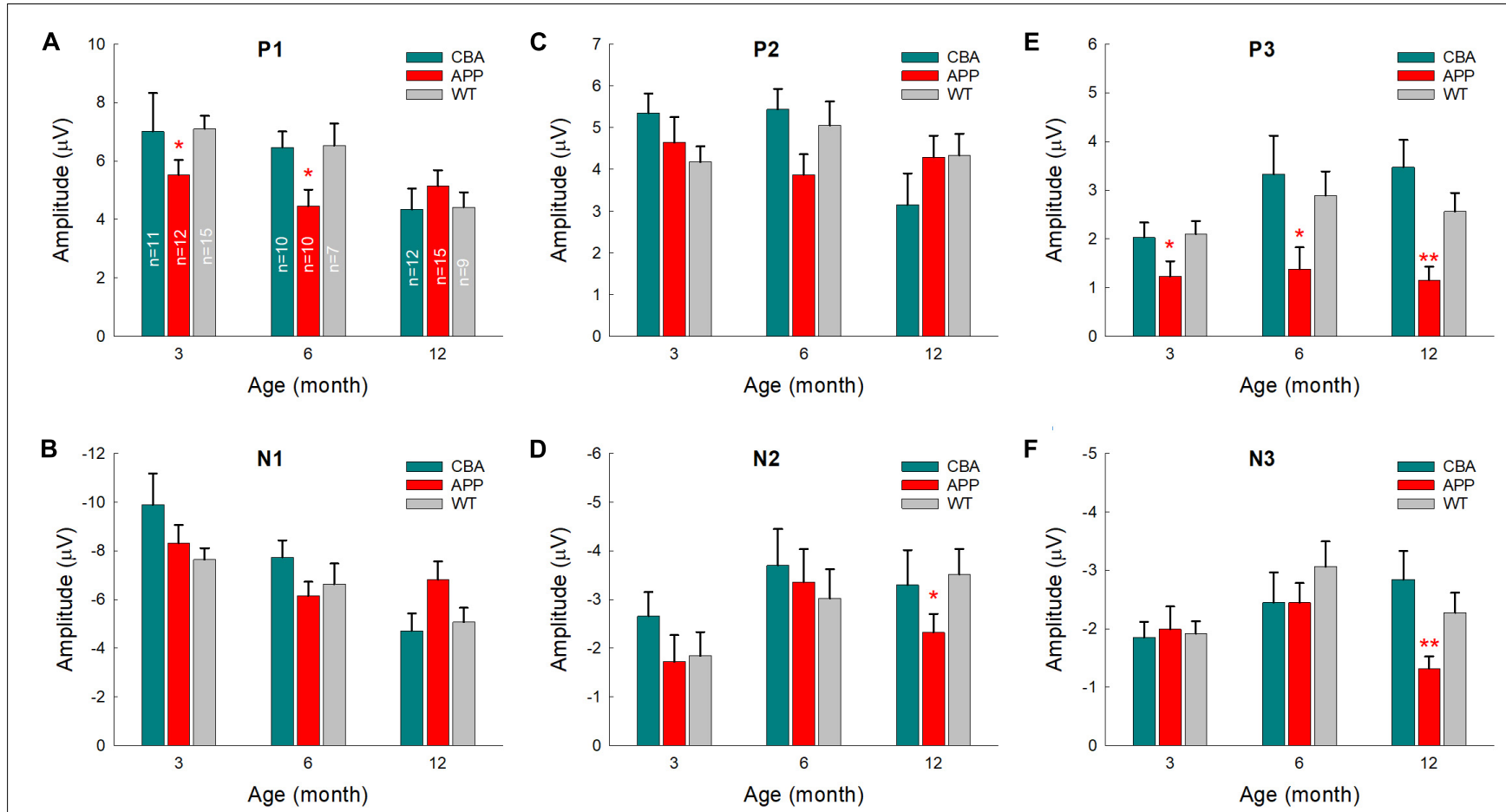

FIGURE 6 | Comparison of AECP changes in CBA/CaJ mice, APP/PS1 AD mice, and WT littermate mice. Peak name is presented at the top middle place in each panel. Animal numbers in each group are presented within the bars in panel (A). Panel (E) shows that peak P3 in the AD mice has a significant reduction in comparison with those in CBA and WT littermate mice. ${ }^{*} p<0.05,{ }^{* *} p<0.01$, one-way ANOVA with a Bonferroni correction.
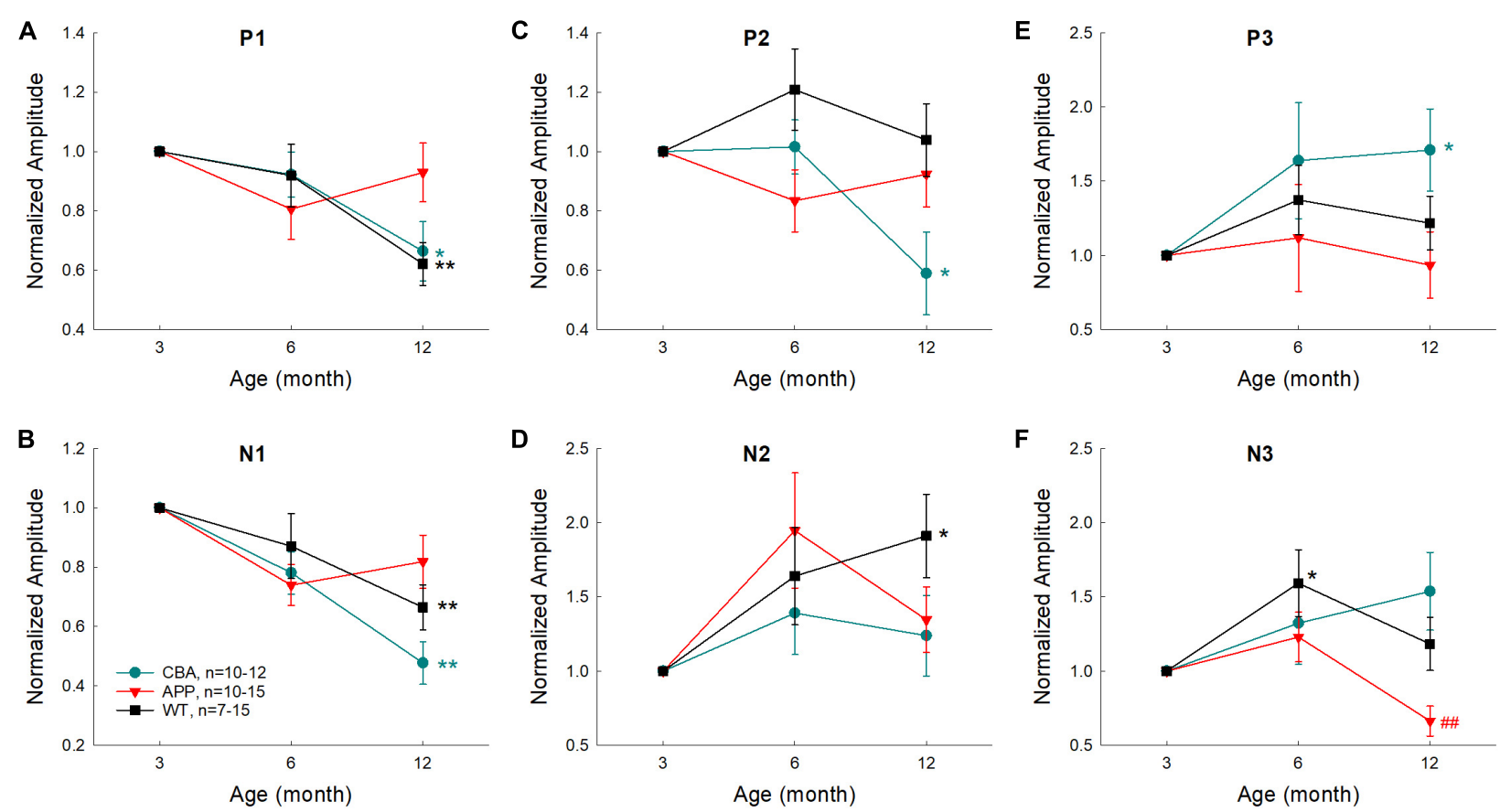

FIGURE 7 | Effects of aging on AECP in CBA/CaJ, APP/PS1 AD, and WT littermate mice. The amplitudes of peaks were normalized to those at 3 months old in each group. Peak name is presented at the top middle place in each panel. $\mathrm{N}$ is the animal number. The statistical $t$-test with two-tailed was performed by comparing data at 3 months or data at 6 months within each group. Early AECP peaks (P1, N1, and P2) in CBA mice and WT littermates are decreased as age increased, while later peaks (N2, P3, and N3) are increased or not changed with aging. However, AD mice have no significant changes in AECP peaks with aging except peak N3 at 12 months old, which is decreased. ${ }^{*} p<0.05,{ }^{* *} p<0.01, t$-test, two-tailed, vs. 3 months; ${ }^{\# \#} p<0.01, t$-test, two-tailed, vs. 6 months. 

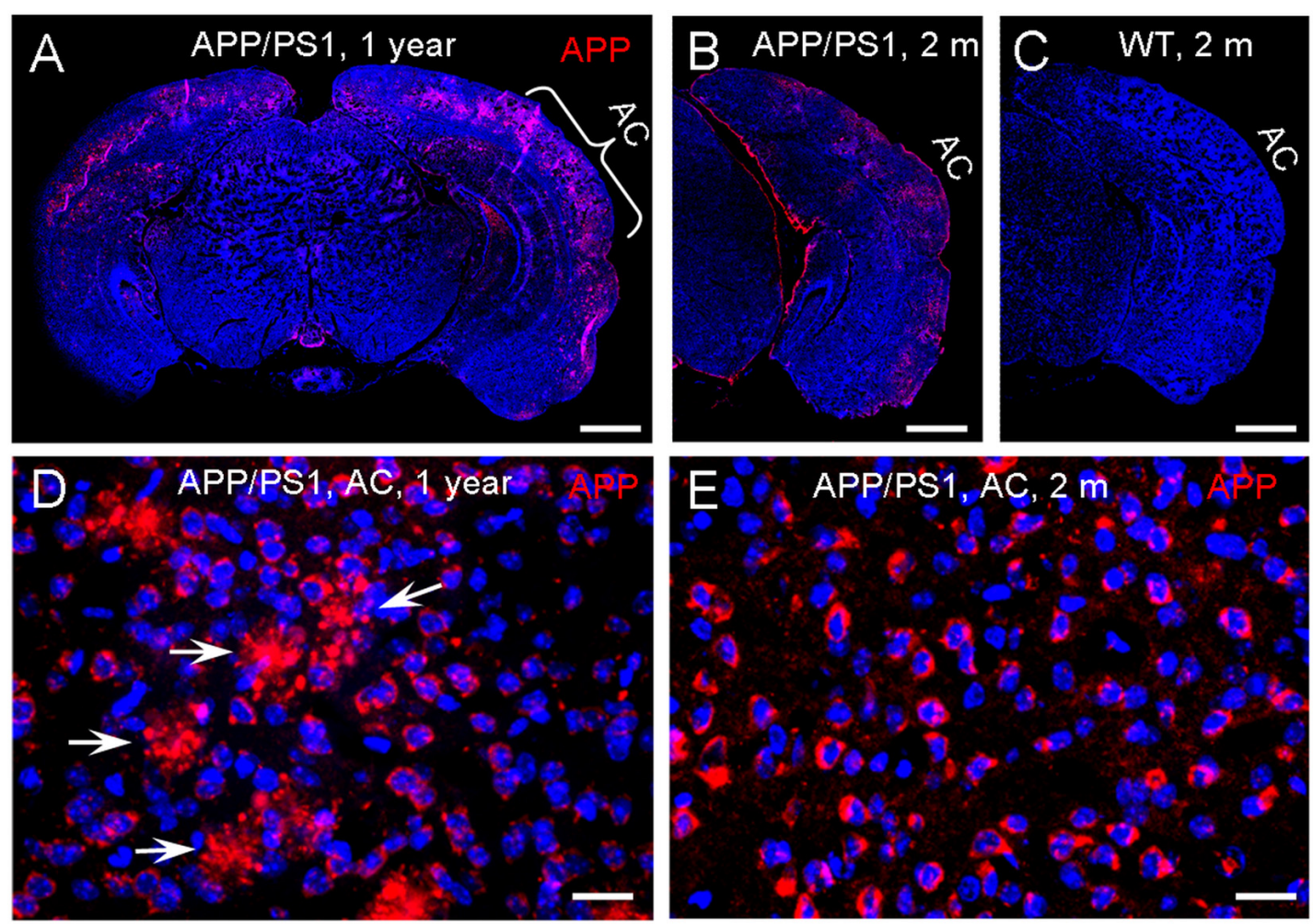

FIGURE 8 | Expression of amyloid precursor protein (APP) in the auditory cortex (AC) in APP/PS1 AD mice. (A-C) Immunofluorescent staining of brain slices in APP/PS1 AD mice and WT mice at the ages of 2 and 12 months old. (D,E) High-magnitude images of positive labeling in the AC area. The positive labeling is visible at the AC area in the APP/PS1 AD mouse at 2 months old. White arrows indicate plaque deposits in the AC area at the 12 months old mouse. Scale bar: $100 \mu \mathrm{m}$ in (A-C), $10 \mu \mathrm{m}$ in (D,E).

with previous reports that memory-based detection and neural activities are recordable in the anesthetized mice (Chen et al., 2015; Kurkela et al., 2018).

The sensory $\mathrm{P} 1$ peak in AD mice appeared smaller than WT mice at young ages ( $<8$ months old) (Figures 3, 4, 6). These data are consistent with previous reports that $\mathrm{AD}$ or amyloid injection could reduce evoked potentials in the AC and other brain cortexes in animal models (Wang et al., 2003; Stoiljkovic et al., 2019; Hidisoglu and Yargicoglu, 2020; Kim et al., 2020). These data also indicated that $\mathrm{AD}$ could result in functional changes in the AC at the early AD stage. It has been reported that APP/PS1 AD mice usually appear typical AD phenotypes, such as spatial learning deficit, by the age of 6-7 months old (Reiserer et al., 2007; Ordóñez-Gutiérrez et al., 2015, 2016). We previously reported that hearing loss measured as $\mathrm{ABR}$ threshold occurred in APP/PS1 AD mice as early as 2-3 months old; ABR wave IV$\mathrm{V}$ were reduced or diminished (Liu et al., 2020). 5xFAD mice also had early onset of deficits in auditory gap detection at 2 months old (Kaylegian et al., 2019; Weible et al., 2020). These data suggest that AECP recording, as well as ABR recording and other hearing function tests, could provide important information for early $\mathrm{AD}$ development and progression. These data also support the concept that auditory sensory and cognitive cortical potentials in $\mathrm{AD}$ persons could be abnormal before $\mathrm{AD}$ becomes apparent (Golob et al., 2009).
In this study, consistent with AECP recording, we found that the accumulation of APP in the AC could be visible in AD mice as early as 2 months old (Figure 8). As age increased, extracellular $\mathrm{A} \beta$-like plaque deposits were also visible (Figure 8D). Previous studies demonstrated that the human brain stem and AC in old $\mathrm{AD}$ patients had $\mathrm{AD}$ pathology including $\mathrm{A} \beta$ plaques, tau protein aggregation, and neural degeneration (Ohm and Braak, 1989; Sinha et al., 1993; Baloyannis et al., 2007, 2009). Our results are consistent with these previous reports and provide further morphological evidence that $\mathrm{AD}$ could occur in the auditory system at the early stage.

In comparison with WT littermates, AECP in AD mice usually appeared small (Figures 3, 4, 6). This is consistent with previous reports that $\mathrm{AD}$ patients had changes in auditoryevoked potentials (Buchwald et al., 1989; Irimajiri et al., 2005; Swords et al., 2018). In particular, the later cognitive peak P3 in $\mathrm{AD}$ mice was diminished (Figures 3, 4, 6), different from the normal aging effect (Figure 7). This is also consistent with a previous report that different from the normal aging effect, cognitive N200 and P300 peaks (i.e., N2 and P3 peaks in mice) in $\mathrm{AD}$ patients were reduced (Morrison et al., 2018). We further found that as age increased, the early peaks ( $\mathrm{P} 1, \mathrm{~N} 1$, and $\mathrm{P} 2$ ) in normal aging CBA mice and WT littermates were decreased but the later peaks (N2, P3, and N3) were increased or not changed (Figures 2, 7), although the 
CBA mice have no significant hearing loss as measured by ABR at least until 1 year old (Zheng et al., 1999). Thus, these data implicate that AECP is dependent not only on brainstem input, in particular, but also on the later peaks in AECP, which are mainly generated by the hippocampus-associated short-term memory (Sur and Sinha, 2009).

The AECP in AD mice had a significant reduction in comparison with WT littermates (Figures 3, 4, 6). However, the AECP in AD mice had no significant changes with aging (Figure 7). This may be due to AECP in AD mice is already reduced and cannot be further reduced as aging increased. In addition, there was no significant delay of AECP peaks in $\mathrm{AD}$ mice (Figure 5), although the latencies of peaks of AECP in normal aging CBA mice were reduced with increased age (Figure 2B). Actually, the latencies of peak P1 and N1 in AD mice at 1-3 months old (Figures 5B-D) and the latencies of $\mathrm{N} 3$ at $10-12$ months old (Figure 5F) were shorter than those in WT mice. This may arise from the fact that the neural interactions in $\mathrm{AD}$ mice may be less than WT mice due to neural degeneration in $\mathrm{AD}$ mice.

At present, more and more $\mathrm{AD}$ mouse models are available and they provide useful animal models to study AD pathological mechanisms, develop therapeutic interventions, and assess the treatment efficiency. However, due to the lack of reliable objective biomarkers, it is still a big challenge to assess cognitive decline in animals, in particular, at the early $\mathrm{AD}$ stage. In this study, we found that later cognitive peak P3 of AECP in AD mice was diminished, different from the normal aging effect (Figures 3, 4, 6, 7). These data suggest that AECP could serve as an objective recording to assess cognitive decline in mice. This will greatly facilitate $\mathrm{AD}$ animal studies.

In this study, we reported a new method by using subdermal needle electrodes with the same electrode configuration as $\mathrm{ABR}$ recording to record $\mathrm{AECP}$ in anesthetized mice. The

\section{REFERENCES}

Baloyannis, S. J., Costa, V., Mauroudis, I., Psaroulis, D., Manolides, S. L., and Manolides, L. S. (2007). Dendritic and spinal pathology in the acoustic cortex in Alzheimer's disease: morphological and morphometric estimation by Golgi technique and electron microscopy. Acta Otolaryngol. 127, 351-354. doi: 10. 1080/00016480601126986

Baloyannis, S. J., Mauroudis, I., Manolides, S. L., and Manolides, L. S. (2009). Synaptic alterations in the medial geniculate bodies and the inferior colliculi in Alzheimer's disease: a Golgi and electron microscope study. Acta Otolaryngol. 129, 416-418. doi: 10.1080/00016480802579074

Buchwald, J. S., Erwin, R. J., Read, S., Van Lancker, D., and Cummings, J. L. (1989). Midlatency auditory evoked responses: differential abnormality of P1 in Alzheimer's disease. Electroencephalogr. Clin. Neurophysiol. 74, 378-384. doi: 10.1016/0168-5597(89)90005-1

Chen, I. W., Helmchen, F., and Lütcke, H. (2015). Specific Early and Late OddballEvoked Responses in Excitatory and Inhibitory Neurons of Mouse Auditory Cortex. J. Neurosci. 35, 12560-12573. doi: 10.1523/JNEUROSCI.2240-15.2015

Gates, G. A., Beiser, A., Rees, T. S., D'Agostino, R. B., and Wolf, P. A. (2002). Central auditory dysfunction may precede the onset of clinical dementia in people with probable Alzheimer's disease. J. Am. Geriatr. Soc. 50, 482-488. doi: 10.1046/j.1532-5415.2002.50114.x

Golob, E. J., Ringman, J. M., Irimajiri, R., Bright, S., Schaffer, B., Medina, L. D., et al. (2009). Cortical event-related potentials in preclinical familial Alzheimer disease. Neurology 73, 1649-1655. doi: 10.1212/wnl.0b013e3181c1de77 recorded AECP was similar to AECP recorded from humans, had both early sensory and later cognitive peaks, and showed distinct response patterns in $\mathrm{AD}$ mice from normal aging effect (Figures 1-3, 6, 7). These data suggest that hearing functional tests, including AECP recording, could serve as simple, non-invasive, repeatable biomarkers to assess $\mathrm{AD}$, and ADRD development and progression.

\section{DATA AVAILABILITY STATEMENT}

The raw data supporting the conclusions of this article will be made available by the authors, without undue reservation.

\section{ETHICS STATEMENT}

The animal study was reviewed and approved by the University of Kentucky Animal Care and Use Committee (approved protocol: UK\#2020-3524).

\section{AUTHOR CONTRIBUTIONS}

$\mathrm{H}-\mathrm{BZ}$ conceived the general framework of this study and wrote the manuscript. LM, L-ML, KC, and $\mathrm{H}-\mathrm{BZ}$ performed the experiments. LM, L-ML, and $\mathrm{H}-\mathrm{BZ}$ analyzed the data. All authors reviewed the manuscript and provided the input.

\section{FUNDING}

This work was supported by the NIH R01 DC 017025 and AD supplement DC 017025-01S1 to H-BZ.

Griffiths, T. D., Lad, M., Kumar, S., Holmes, E., McMurray, B., Maguire, E. A., et al. (2020). How can hearing loss cause dementia? Neuron 108, 401-412. doi: 10.1016/j.neuron.2020.08.003

Gurevicius, K., Lipponen, A., and Tanila, H. (2013). Increased cortical and thalamic excitability in freely moving APPswe/PS1dE9 mice modeling epileptic activity associated with Alzheimer's disease. Cereb. Cortex 23, 1148-1158. doi: 10.1093/ cercor/bhs105

Hebert, L. E., Weuve, J., Scherr, P. A., and Evans, D. A. (2013). Alzheimer disease in the United States (2010-2050) estimated using the 2010 census. Neurology 80 , 1778-1783. doi: 10.1212/wnl.0b013e31828726f5

Hidisoglu, E., and Yargicoglu, P. (2020). Auditory evoked potentials might have the potential to serve as early indicators related to amyloid beta peptide toxicity. Adv. Med. Sci. 65, 223-232. doi: 10.1016/j.advms.2020.02.001

Irimajiri, R., Golob, E. J., and Starr, A. (2005). Auditory brain-stem, middleand long-latency evoked potentials in mild cognitive impairment. Clin. Neurophysiol. 116, 1918-1929. doi: 10.1016/j.clinph.2005.04.010

Kaylegian, K., Stebritz, A. J., Weible, A. P., and Wehr, M. (2019). 5XFAD Mice Show Early Onset Gap Detection Deficits. Front. Aging Neurosci. 11:66. doi: 10.3389/fnagi.2019.00066

Kim, B., Shin, J., Kim, Y., and Choi, J. H. (2020). Destruction of ERP responses to deviance in an auditory oddball paradigm in amyloid infusion mice with memory deficits. PLoS One 15:e0230277. doi: 10.1371/journal.pone.023 0277

Kurkela, J. L. O., Lipponen, A., Kyläheiko, I., and Astikainen, P. (2018). Electrophysiological evidence of memory-based detection of auditory regularity 
violations in anesthetized mice. Sci. Rep. 8:3027. doi: 10.1038/s41598-01821411-Z

Liu, Y., Fang, S., Liu, L. M., Zhu, Y., Li, C. R., Chen, K., et al. (2020). Hearing loss is an early biomarker in APP/PS1 Alzheimer's disease mice. Neurosci. Lett. 717:134705. doi: 10.1016/j.neulet.2019.134705

Morrison, C., Rabipour, S., Knoefel, F., Sheppard, C., and Taler, V. (2018). Auditory event-related potentials in mild cognitive impairment and Alzheimer's disease. Curr. Alzheimer Res. 15, 702-715. doi: 10.2174/1567205015666180123123209

Murphy, C. (2019). Olfactory and other sensory impairments in Alzheimer disease. Nat. Rev. Neurol. 15, 11-24. doi: 10.1038/s41582-018-0097-5

Ohm, T. G., and Braak, H. (1989). Auditory brainstem nuclei in Alzheimer's disease. Neurosci. Lett. 96, 60-63. doi: 10.1016/0304-3940(89)90243-7

Ordóñez-Gutiérrez, L., Antón, M., and Wandosell, F. (2015). Peripheral amyloid levels present gender differences associated with aging in A $\beta P P / P S 1$ mice. J. Alzheimers Dis. 44, 1063-1068. doi: 10.3233/JAD- 141158

Ordóñez-Gutiérrez, L., Fernandez-Perez, I., Herrera, J. L., Anton, M., BenitoCuesta, I., and Wandosell, F. (2016). A $\beta$ PP/PS1 transgenic mice show sex differences in the cerebellum associated with aging. J. Alzheimers Dis. 54, 645-656. doi: 10.3233/jad-160572

Patterson, C. (2018). The state of the art of dementia research: new frontiers. Word Alzheimer Report 2018. London: Alzheimer's Disease International.

Reiserer, R. S., Harrison, F. E., Syverud, D. C., and McDonald, M. P. (2007). Impaired spatial learning in the APP + PSEN1DeltaE9 bigenic mouse model of Alzheimer's disease. Genes Brain Behav. 6, 54-65. doi: 10.1111/j.1601-183x. 2006.00221.x

Rochoy, M., Rivas, V., Chazard, E., Decarpentry, E., Saudemont, G., Hazard, P. A., et al. (2019). Factors associated with Alzheimer's disease: an overview of reviews. J. Prev. Alzheimers Dis. 6, 121-134. doi: 10.14283/jpad.2019.7

Sinha, U. K., Hollen, K. M., Rodriguez, R., and Miller, C. A. (1993). Auditory system degeneration in Alzheimer's disease. Neurology 43, 779-785. doi: 10.1212/wnl. 43.4.779

Stoiljkovic, M., Kelley, C., Stutz, B., Horvath, T. L., and Hajós, M. (2019). Altered cortical and hippocampal excitability in TgF344-AD rats modeling Alzheimer's disease pathology. Cereb. Cortex 29, 2716-2727. doi: 10.1093/cercor/bhy140

Sur, S., and Sinha, V. K. (2009). Event-related potential: an overview. Ind. Psychiatry J. 18, 70-73. doi: 10.4103/0972-6748.57865
Swords, G. M., Nguyen, L. T., Mudar, R. A., and Llano, D. A. (2018). Auditory system dysfunction in Alzheimer disease and its prodromal states: a review. Ageing Res. Rev. 44, 49-59. doi: 10.1016/j.arr.2018.04.001

Wang, J., Ikonen, S., Gurevicius, K., Van Groen, T., and Tanila, H. (2003). Altered auditory-evoked potentials in mice carrying mutated human amyloid precursor protein and presenilin-1 transgenes. Neuroscience 116, 511-517. doi: 10.1016/ s0306-4522(02)00714-5

Wang, X. H., Streeter, M., Liu, Y. P., and Zhao, H. B. (2009). Identification and characterization of pannexin expression in the mammalian cochlea. J. Comp. Neurol. 512, 336-346. doi: 10.1002/cne.21898

Weible, A. P., Stebritz, A. J., and Wehr, M. (2020). 5XFAD mice show early-onset gap encoding deficits in the auditory cortex. Neurobiol. Aging 94, 101-110. doi: 10.1016/j.neurobiolaging.2020.05.013

Zhao, H. B., and Yu, N. (2006). Distinct and gradient distributions of connexin 26 and connexin30 in the cochlear sensory epithelium of guinea pigs. J. Comp. Neurol. 499, 506-518. doi: 10.1002/cne.21113

Zheng, Q. Y., Johnson, K. R., and Erway, L. C. (1999). Assessment of hearing in 80 inbred strains of mice by ABR threshold analyses. Hear. Res. 130, 94-107. doi: 10.1016/s0378-5955(99)00003-9

Conflict of Interest: The authors declare that the research was conducted in the absence of any commercial or financial relationships that could be construed as a potential conflict of interest.

Publisher's Note: All claims expressed in this article are solely those of the authors and do not necessarily represent those of their affiliated organizations, or those of the publisher, the editors and the reviewers. Any product that may be evaluated in this article, or claim that may be made by its manufacturer, is not guaranteed or endorsed by the publisher.

Copyright (c) $2021 \mathrm{Mei}$, Liu, Chen and Zhao. This is an open-access article distributed under the terms of the Creative Commons Attribution License (CC BY). The use, distribution or reproduction in other forums is permitted, provided the original author(s) and the copyright owner(s) are credited and that the original publication in this journal is cited, in accordance with accepted academic practice. No use, distribution or reproduction is permitted which does not comply with these terms. 